\title{
¿Qué espera el clínico del informe radiológico?
}

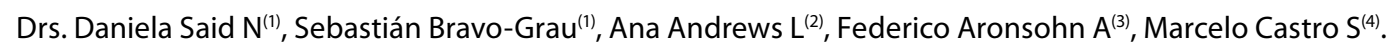

1. Residente de Radiología, Facultad de Medicina, Universidad de los Andes - Santiago, Chile.

2. Residente de Radiología, Facultad de Medicina, Universidad de Chile - Santiago, Chile.

3. Médico-cirujano, Facultad de Medicina, Universidad de los Andes - Santiago, Chile.

4. Radiólogo, Departamento de Diagnóstico por Imágenes, Hospital Militar de Santiago - Santiago, Chile.

What does the clinician expect from the radiological report?

Abstract: Introduction:The written radiology report is the most relevant component of any radiological examination. Few studies about referring clinicians opinion concerning structure and contents of radiology reports have been conducted. Objective: To investigate which radiology report issues are significant to the clinician. Methodology: One hundred and sixty-four physicians, among surgeons, internists, residents, and general practitioners were surveyed. Results: According to surveyed clinicians the most important element in any radiologic examination is the reporting radiologist (67.7\%); $93.9 \%$ of them expect the radiology report to address the referral diagnosis; $95.7 \%$ expects the report to present a conclusion, whilst $73 \%$ of referring clinicians consider ultrasonography as the imaging procedure that commonly exhibits more errors. Surgeons, generally understimate radiologists recommendations for complementary studies. Conclusion: An experienced reporting radiologist is considered the most significant element of any imaging procedure. The radiology report should be problem-oriented (answering clinical question), and contain a conclusion, which should begin by answering the clinician's concerns. General practitioners, internists and residents value further studies reccommended by radiologists.

Key words: Communication, Diagnostic imaging, Physicians, Radiology, Radiological report.

Resumen: Introducción. El informe es lo más relevante de un examen radiológico. Escasas publicaciones han estudiado la opinión del clínico. Objetivo. Establecer qué aspectos del informe son relevantes para el clínico. Metodología. Se aplicó una encuesta a cirujanos, internistas, residentes y médicos generales. Resultados. Se encuestó a 164 médicos. Al pedir un examen radiológico, lo más relevante $(67,7 \%)$ fue el radiólogo informante; 93,9\% considera importante que se haga referencia al diagnóstico de derivación; 95,7\% estima que debe haber conclusión. El $73 \%$ cree que el examen en el que se cometen más errores es la ecotomografía. Los cirujanos son los que menos valoran las recomendaciones de estudios posteriores. Conclusión. El radiólogo que informa es considerado lo más importante en un examen. El informe debe hacer referencia al diagnóstico, tener conclusión, la que debe comenzar respondiendo la inquietud del clínico. Los médicos generales, internistas y residentes valoran que se sugieran estudios complementarios.

Palabras clave: Clínicos, Comunicación, Imaginología diagnóstica, Informe radiológico, Médicos, Radiología.

Said D, et al. ¿Qué espera el clínico del informe radiológico? Rev Chil Radiol 2012; 18(3): 111-116.

Correspondencia: Dra. Daniela Said N. / dani_said@hotmail.com

Trabajo recibido el 20 de agosto de 2012, aceptado para publicación el 04 del septiembre de 2012.

Introducción

El informe radiológico constituye uno de los aspectos más relevantes de un examen imaginológico, siendo la principal fuente de comunicación entre el radiólogo y el médico clínico. Pese a su importancia es el aspecto menos estandarizado de un examen imaginológico.

En los programas de formación de especialista no se enseña de forma explícita como informar o reportar los hallazgos patológicos, qué priorizar y qué aspectos les son relevantes para el clínico. Por otra parte, en pregrado no se enseña a los futuros médicos a cómo realizar una solicitud de examen. Estos hechos dificultan la comunicación entre el radiólogo y el clínico, determinando entre otras cosas, mayores costos para pacientes e instituciones. De más está decir el rol médico legal del informe, como constituyente del acto médico en el caso del radiólogo ${ }^{(1)}$.

Es el médico radiólogo el responsable del informe emitido, el cual debe ser preciso, conciso y pertinente, 
que responda claramente a las preguntas del clínico y que ayude al diagnóstico y a la resolución de la enfermedad.

En la literatura anglosajona hay opiniones de expertos que entregan recomendaciones de cómo debería ser un informe ${ }^{(2)}$. Publicaciones españolas también han revisado distintos aspectos del infor$\mathrm{me}^{(3,4)}$. A nivel nacional se ha discutido el rol que tiene la construcción del informe, en cuánto a los errores en radiología(5) y se ha estudiado la influencia de las circunstancias clínicas en la percepción de los informes por los médicos solicitantes( ${ }^{(6)}$.

A pesar de todo esto, prácticamente no existen publicaciones nacionales respecto de cómo debe ser realizado el informe, para satisfacer la inquietud del clínico, ni cuáles son los elementos del informe radiológico que este último más valora.

Dada la trascendencia del informe para el paciente y la toma de decisiones clínicas, consideramos de importancia conocer lo que el médico clínico espera y opina de la estructura y contenido de éste.

Los objetivos de este estudio fueron: conocer qué espera el clínico de un informe radiológico. Específicamente, estudiar las preferencias de los clínicos respecto a los aspectos que debieran incluir la descripción y conclusión de un informe radiológico, además, identificar cuáles son las características que más valoran los clínicos de un informe radiológico. Secundariamente, intentar establecer diferencias entre los grupos de médicos según especialidad: cirujanos, internistas, residentes y médicos generales.

\section{Materiales y métodos}

Se realizó un estudio descriptivo transversal, empleando una muestra intencionada de sujetos voluntarios. Durante el primer semestre del año 2011, se aplicó una encuesta escrita a médicos especialistas en cirugía y medicina interna, médicos generales y becados o residentes de cirugía y medicina interna. Esta encuesta incluye 5 preguntas de alternativas y 2 preguntas en la que se les solicitó a los médicos evaluar determinados aspectos del informe radiológico y de la conclusión del informe (anexo 1).

Se les solicitó que respondieran su especialidad, sexo, años de ejercicio profesional e institución donde se desempeñan. Considerando un informe radiológico estándar, se les pidió que analizaran diferentes aspectos de éste y de su conclusión, anotando si les parecía importante o irrelevante determinadas características. También se preguntó con qué frecuencia entregan información clínica al solicitar un examen radiológico; a qué atribuyen los errores en un informe, qué conducta toman frente a un examen insatisfactorio y qué factores consideran antes de pedir un examen imaginológico.

Para el análisis estadístico se utilizó prueba chi- cuadrado de Pearson, y prueba exacta de Fisher. Se consideró como significativo un P-valor menor a 0,05.

\section{Resultados}

Se encuestó a 164 médicos de los cuales el $17 \%$ eran residentes o becados; $29 \%$ médicos generales; $21 \%$ cirujanos; y $33 \%$ internistas. El $74 \%$ fue de sexo masculino y el $26 \%$ femenino. Respecto a la institución donde se desempeñan, el $86 \%$ trabaja en hospital y/o clínica y el $14 \%$ en un centro de atención primaria. En relación a los años de experiencia el 54\% tenía más de 10 años de ejercicio profesional.

El aspecto más relevante al pedir un examen imaginológico fue en primer lugar el radiólogo que lo va a informar (68\%) y en segundo lugar el centro donde se efectuará el examen (25\%) (Figura 1).

Al consultar a los médicos clínicos la importancia que le daban a diferentes aspectos del informe: el 93,9\% considera importante que el radiólogo haga referencia al diagnóstico clínico por el cual se solicitó el examen; el $87,8 \%$ piensa que es importante que se mencione la patología banal encontrada; y el $85,4 \%$ considera importante que se respete la ortografía y semántica (Tabla I). No se encontraron diferencias estadísticamente significativas en estas respuestas al dividirlas por especialidad.

Respecto de las preferencias de los clínicos en relación a la conclusión del informe radiológico: el $95,7 \%$ de los médicos encuestados estiman que el informe debe tener una conclusión final o impresión diagnóstica; y al 78,8\% le gustaría que la primera impresión diagnóstica en la conclusión sea la respuesta a la solicitud del examen (Tabla II).

Se analizaron las respuestas en relación a determinados aspectos de la conclusión del informe radiológico, por grupos de médicos: médicos generales, internistas, cirujanos y becados. Se encontraron diferencias en la preferencia respecto de si deseaban sugerencias de exámenes complementarios en la conclusión, siendo los cirujanos quienes menos favorecen esto (Tabla III). En este caso existen diferencias estadísticamente significativas entre los cirujanos y el resto de los médicos.

Se les preguntó en qué porcentaje de las solicitudes de exámenes radiológicos, aportaban información clínica. Un $67 \%$ manifiesta entregar información clínica en más del $75 \%$ de sus peticiones. Existe una diferencia estadísticamente significativa entre los médicos generales y el resto de los médicos. Los médicos generales aportan menos datos clínicos que el resto de los médicos encuestados $(\mathrm{P}<0,05)$ (Figura 2).

En cuanto a la apreciación de los clínicos acerca de la causa más frecuente de error del radiólogo: el 58\% lo atribuyó a falta de expertise del radiólogo y el $32 \%$ a la falta de antecedentes clínicos aportados en la orden médica. No hubo 
ANEXO 1

Encuesta: INFORMES RADIOLÓGICOS

Con el objeto de mejorar la calidad de los informes radiológicos favor complete la siguiente encuesta:

Especialidad: ( ) Medicina General ( ) Cirujano ( ) Becado ( ) Otra. Mencionar

Sexo: ( ) F ( ) M. Institución donde trabaja Años de ejercicio profesional

1. En un examen radiológico, ¿Qué considera Ud, más importante? (marque sólo una alternativa).

a) Centro donde se hizo el examen

b) Radiólogo informante

c) Costo del examen

d) Formato en como se entrega el examen (Placa, CD, impresión en papel)

2. Marque con X el grado de importancia de cada uno de los siguientes aspectos del informe radiológico.

Descripción de órganos no patológicos

Mención de patología banal incidentalmente encontrada

Informe radiológico breve

Descripción de la técnica del examen radiológico

El radiólogo haga referencia al diagnóstico clínico por el cual se solicitó el examen

Se respete la ortografía y semántica

3. Respecto a la CONCLUSIÓN del informe radiológico, le gustaría que (Marque con una X):

La primera impresión sea la respuesta a la pregunta formulada en su solicitud

Se sugieran exámenes complementarios

Incluya las patologías banales encontradas incidentalmente

Es necesario que tenga conclusión (a parte de descripción)

4. ¿Con qué frecuencia aporta Ud., antecedentes clínicos a la orden de solicitud de un examen de radiología

(Marque sólo una alternativa)
a) $76-100 \%$
b) $51-75 \%$
c) $25-50 \%$
d) $<25 \%$

5. ¿Cuál cree Ud., que es la principal causa de equívoco del radiológo? (Marque SÓLO una):
a) Falta de diagnóstico de derivación o antecedentes clínicos aportados en la orden médica
b) Examen mal indicado
c) Equipos radiológicos obsoletos o de mala calidad
d) Expertise del radiólogo informante

6. ¿En qué examen cree Ud., que se cometen más errores? (Marque SÓLO una):
a) Radiografía
b) Ecotomografía
c) Tomografía computada
d) Resonancia magnética

7. Frente a un examen radiológico que NO le entrega la información que Ud. requiere o bien no le satisfizo respecto a su calidad, ¿que conducta toma? (Marque sólo una alternativa)
a) Le comenta al paciente y le dice que el examen está mal hecho y que debe quejarse
b) Repite el examen
c) Se comunica con el radiólogo que lo informó
d) Le mestra el examen a otro radiólogo para ver que opina
e) Pide otros exámenes

diferencias estadísticamente significativas entre diferentes especialidades (Figura 3).

En el sexto ítem se preguntó a los encuestados sobre cuál es el examen que creen es donde más errores se cometen. El 73\% respondió la alternativa correspondiente a ecotomografía, seguido por la radiología simple. Sin diferencias entre especiali- dades (Figura 4).

Finalmente, frente a un informe radiológico no satisfactorio, el $43,8 \%$ dice comunicarse con el radiólogo que lo informó; el $28,7 \%$ lo discute con otro radiólogo; y el $26,1 \%$ lo repite o realiza otro examen. No existen tampoco diferencias entre las especialidades (Figura 5). 


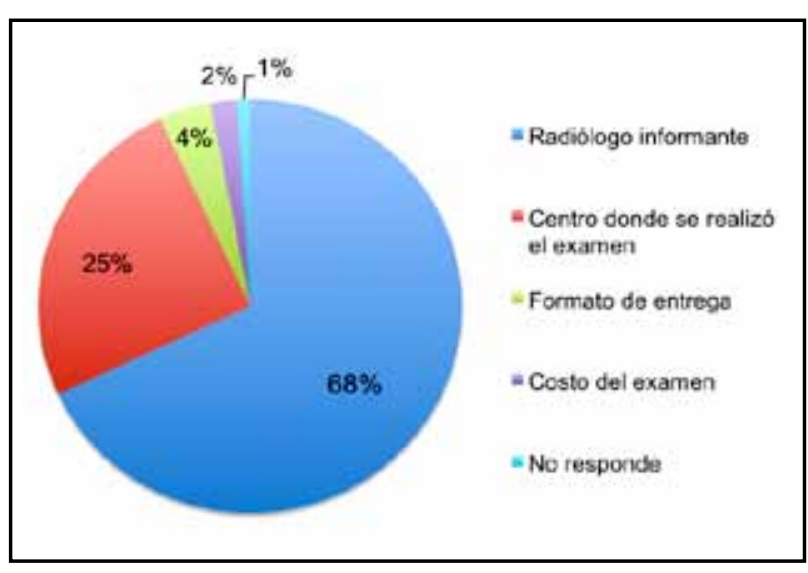

Figura 1. Qué considera el médico clínico más importante en un examen radiológico.

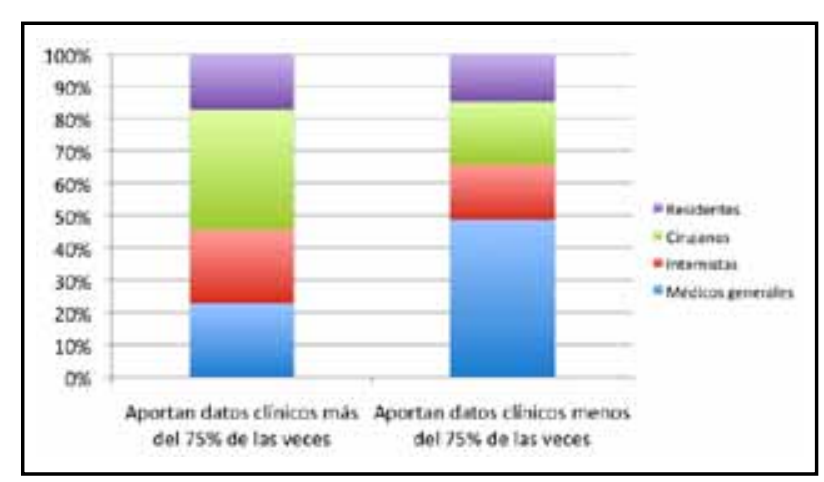

Figura 2. Proporción de médicos según especialidad y formación, que declaran aportar información clínica al solicitar exámenes radiológicos; más del $75 \%$ de las veces y menos del $75 \%$ de las veces.

Tabla I. Importancia otorgada a cada uno de los siguientes aspectos del informe radiológico. Porcentaje de respuestas en cada categoría.

Se haga referencia al diagnóstico por el cual se solicitó el examen Mención de patología banal incidentalmente encontrada Se respete la ortografía y semántica Descripción de órganos no patológicos Informe radiológico breve Descripción de la técnica del examen radiológico

\begin{tabular}{|crc|}
\hline Importante & Irrelevante & No responde \\
\hline $93,0 \%$ & $6,1 \%$ & $0,0 \%$ \\
$87,8 \%$ & $10,4 \%$ & $1,8 \%$ \\
$85,4 \%$ & $14,6 \%$ & $0,0 \%$ \\
$80,5 \%$ & $18,3 \%$ & $1,2 \%$ \\
$62,8 \%$ & $33,5 \%$ & $3,7 \%$ \\
$60,4 \%$ & $37,8 \%$ & $1,8 \%$ \\
\hline
\end{tabular}

Tabla II. Preferencias respecto a determinados aspectos de la conclusión del informe radiológico. Porcentaje de respuesta de los encuestados.

Respecto a la conclusión del informe radiológico, a usted le gustaría que:

Es necesario que tenga conclusión (además de descripción) La primera impresión sea la respuesta a la pregunta formulada en su solicitud Incluya las patologías banales encontradas incidentalmente Se sugieran exámenes complementarios

\begin{tabular}{|c|c|}
\hline \multicolumn{1}{|c|}{ Sí } & $\begin{array}{c}\text { No } \\
\text { o indeferente }\end{array}$ \\
\hline $95,7 \%$ & $3,6 \%$ \\
$78,7 \%$ & $21,3 \%$ \\
$62,8 \%$ & $36,0 \%$ \\
$58,5 \%$ & $40,3 \%$ \\
\hline
\end{tabular}

Tabla III. Preferencias por los distintos grupos de médicos encuestados, según especialidad y formación, respecto a sugerencia de exámenes complementarios.

Le gustaría que se sugieran exámenes complementarios en la conclusión:

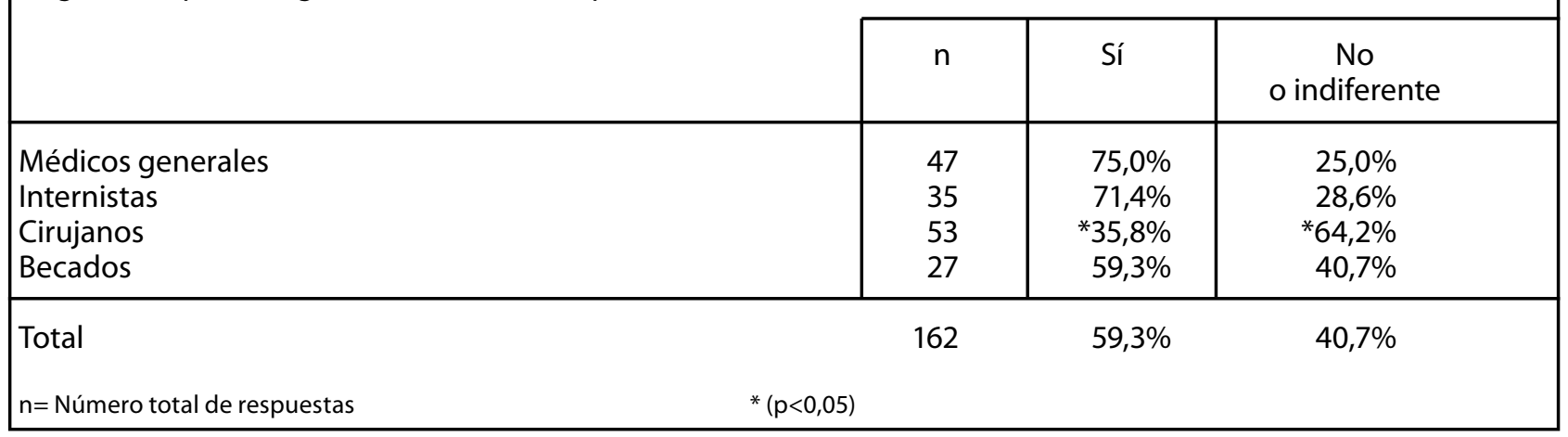




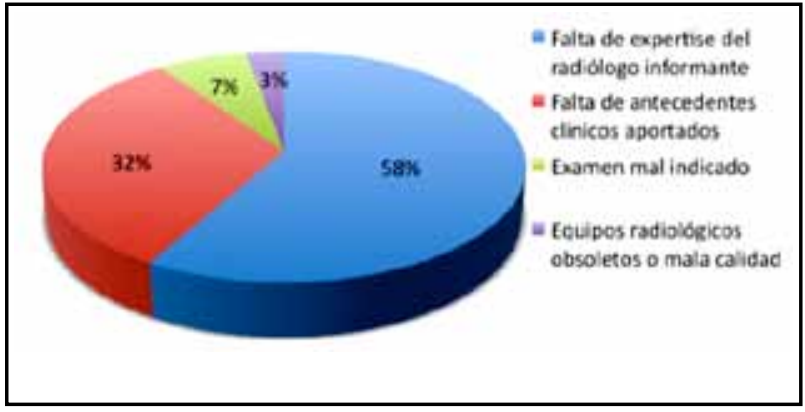

Figura 3. Apreciación del clínico en cuánto a la principal causa de error del radiólogo.

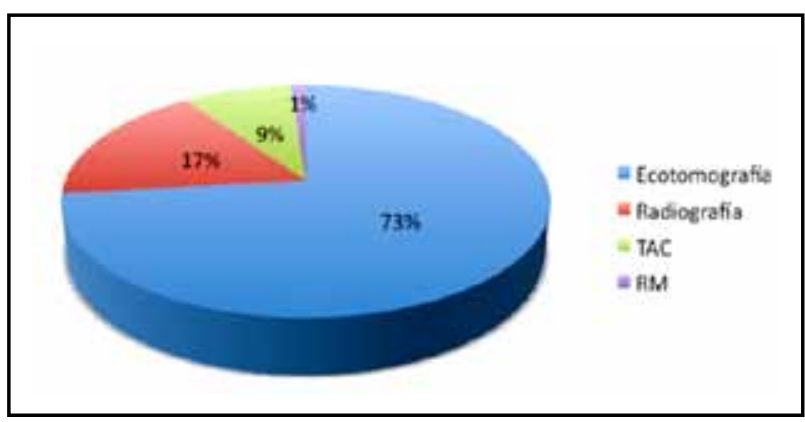

Figura 4. Apreciación del clínico en cuánto al examen radiológico en el que se cometen más errores.

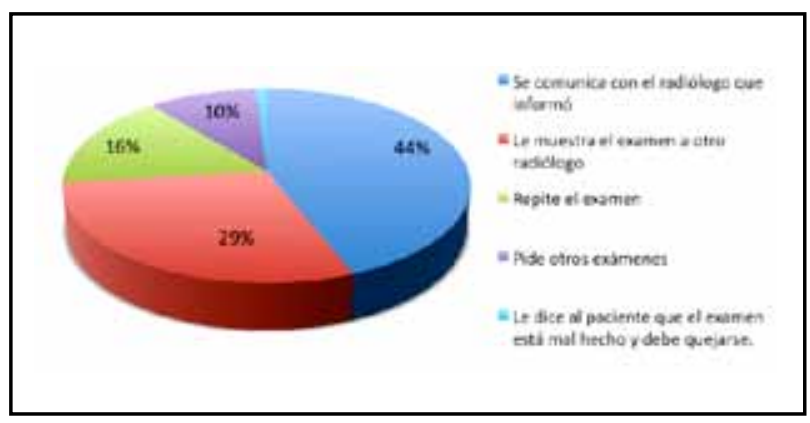

Figura 5. Conducta de los médicos clínicos frente a un examen no satisfactorio.

\section{Discusión}

Según la opinión del clínico la experiencia del radiólogo informante es el aspecto que mayor valor le da al momento de solicitar un examen radiológico. Además, es el radiólogo el principal responsable si el informe no aporta la información que el clínico requiere.

El radiólogo debe hacer referencia al diagnóstico clínico por el cual fue derivado el paciente. Como lo muestra un estudio europeo: la referencia explícita del órgano o estructura en cuestión, incluso siendo normales, se consideraría por los clínicos como evidencia de que el radiólogo si observó las estructuras anatómicas vinculadas con la observación diagnóstica ${ }^{(7)}$.

Además, un alto porcentaje de los encuestados considera importante mencionar la patología banal incidentalmente encontrada, especialmente los médicos generales. Más aun, no es prioridad para los clínicos que el informe sea breve. Lo anterior es concordante con un estudio nacional ${ }^{(6)}$ y con lo comunicado por otros autores asiáticos ${ }^{(8)}$ : habría una mayor preferencia por informes detallados, por parte de los clínicos.

Los encuestados en este estudio son categóricos en señalar que el informe radiológico requiere tener conclusión y esta última debe comenzar respondiendo la inquietud por la cual es solicitado el examen. Los médicos generales e internistas son los que más valoran la sugerencia de exámenes complementarios, no así los cirujanos. En un hospital universitario de nuestro país, observaron este hecho, de que los clínicos eran reticentes a las recomendaciones, sin reportar diferencias por especialidad ${ }^{(6)}$. Un estudio asiático evidenció que 70,5\% de sus clínicos aprueba la inclusión de recomendaciones en el informe ${ }^{(8)}$. Este aspecto parece variar significativamente en cada centro y depende de las respectivas idiosincrasias.

Bosmans et al, en un estudio reciente, entregó evidencias en relación a la importancia que le otorgan clínicos y radiólogos, transversalmente, a la entrega de información clínica al solicitar imágenes ${ }^{(7)}$. En nuestra investigación, una proporción mayoritaria de los encuestados dice aportar antecedentes clínicos en más del $75 \%$ de las veces en que solicita un examen imaginológico. Los médicos generales son los que menos información entregan.

Los clínicos encuestados perciben la ecotomografía como el examen más susceptible de error; probablemente por ser operador dependiente. Con respecto a las causas de error en un examen imaginológico, la experiencia o habilidad del radiólogo y la falta de información clínica, son los dos principales condicionantes, según la opinión de los encuestados. Diferentes actitudes toman los clínicos frente a informes no satisfactorios. Es importante destacar que en el $73 \%$ es un radiólogo al cual se acude para solicitar ayuda.

Por motivos prácticos la encuesta confeccionada se centra en determinadas inquietudes y características del informe radiológico y del proceso de solicitud de exámenes imaginológicos. Las preguntas eran de tipo cerrado, existiendo un número determinado de alternativas para su respuesta. Esto limita las opciones de los encuestados y se debe considerar al sopesar nuestros hallazgos. En este sentido, es de interés la confección de una encuesta que cumpla con los criterios científicos de validez interna y externa para nuestra población de médicos clínicos nacionales, que sea de una extensión adecuada para su aplicación, comparable en el tiempo y que evalúe los aspectos más 
importantes y críticos para los radiólogos, clínicos y pacientes.

Como se mencionó, este estudio corresponde a una muestra intencionada de sujetos voluntarios. Por lo tanto, corresponde a una exploración inicial en relación a las características que espera el clínico con respecto al informe radiológico. No pretende ser representativa de nuestra población. Similares evaluaciones se deberían realizar en los respectivos centros, para conocer las expectativas de sus clínicos. Es de interés, además, realizar estudios posteriores con estrategias de muestreo, los cuales permitan que los resultados sean extrapolables a nuestro entorno nacional o a una determinada unidad espacial.

\section{Bibliografía}

1. Ritz J. La reforma procesal penal chilena: Nuevo marco médico-legal del ejercicio de la radiología. Rev Chil Radiol 2005; 11: 142-149.
2. Hall FM. Language of the radiology report. Primer for residents and wayward radiologists. AJR Am J Roentgenol 2000; 175: 1239-1242.

3. Tardáguila F, Martí-Bonmatí L, Bonmatí J. El informe radiológico: filosofía general (I). Radiología 2004; 46: 195-198.

4. Martí-Bonmatí L, Tardáguila F, Bonmatí J. El informe radiológico: Estilo y contenido (II). Radiología 2004; 46: 199-202.

5. Ortega D, García C. Prevención de riesgo en radiología: El error y el radiólogo. Rev Chil Radiol 2002; 8: 135-140.

6. Ortega D, Tapia A, Ortega X, Muñoz S. El informe radiológico: la opinión de los clínicos. Rev Chil Radiol 2000; 6: 151-154.

7. Bosmans JML, Weyler JJ, de Schepper AM, Parizel PM. The radiology report as seen by radiologists and referring clinicians: results of the COVER and ROVER surveys. Radiology 2011; 259: 184-195.

8. Dogan N, Varlibas ZN, Erpolat OP. Radiological report: expectations of clinicians. Diagn Interv Radiol 2010; 16: 179-185. 